Published in final edited form as:

Surg Neurol. 2008 July ; 70(1): 36-38. doi:10.1016/j.surneu.2008.02.017.

\title{
Molecular pathology of aneurysms
}

\author{
Mika Niemelä, MD, PhD, Juhana Frösen, MD, PhD, and Juha Hernesniemi, MD, PhD \\ Department of Neurosurgery, Helsinki University Central Hospital, 00260 Helsinki, Finland \\ Reza Dashti, MD \\ Cerraphasa Medical Faculty, University of Istanbul, Aksaray, 34303 Istanbul, Turkey \\ Aarno Palotie, MD, PhD \\ The Wellcome Trust Sanger Institute, Hinxton, Cambridge, CB101 SA, UK, The Finnish Genome \\ Center, 00290 Helsinki, Finland, The Broad Institiute of MIT and Harvard, Cambridge, 02142 MA, \\ USA
}

\begin{abstract}
The most efficient way to reduce the high morbidity and mortality related to subarachnoid hemorrhage (SAH) would be to treat cerebral aneurysms before they rupture. Patients with a family history for aneurysms ( 2 or more first-degree relatives with aneurysms) should be screened with magnetic resonance angiography or computed tomography angiography and new aneurysms found should be treated in advance depending on their size and location and other risk factors. Furthermore, these patients should be advised to avoid smoking and have their possible hypertension be treated even if the primary radiological examinations were found to be negative as so far we have no test available to exclude a gene mutation predisposing these patients to these potentially lethal sacs.
\end{abstract}

Sung-Pil Joo et al address an important topic of the molecular pathology of SAH caused by sporadic saccular cerebral aneurysms. Despite the relatively high prevalence of them and aneurysmal SAH in the Western populations, and especially in the Japanese and Finnish population, little is still known about the molecular mechanisms leading to the rupture of aneurysms. This knowledge is essential for the development of novel tools for the diagnostics of rupture-prone ones and to engineer novel, less invasive therapies to prevent their rupture.

A genetic approach is one way to uncover cellular pathways resulting in aneurysms. Familybased linkage studies have identified chromosomal regions which harbour potential susceptibility genes $[15,16]$. Recently, the first gene convincingly associated with common vascular aneurysms, both abdominal and intracerebral, was reported by an international consortium led by the Icelandic company deCODE genetics (Reykjavik, Iceland) [3]. However, it is clear that this one identified variant explains only a small fraction of the genetic variance in disease susceptibility. Thus many more genes and variants contribute to the pathophysiology of cerebral aneurysms.

The report by Sung-Pil Joo et al in this volume of Surgical Neurology uses a classical candidate gene strategy. They test whether variants in one functionally relevant candidate gene, endoglin, a receptor for transforming growth factor $\beta$, would predispose to the formation and rupture of cerebral aneurysms. Endoglin is a key member in the growth factor signaling cascade that controls the maturation of new blood vessels during angiogenesis [7],

(C) 2008 Elsevier Inc. All rights reserved.

E-mail addresses.mika.niemela@hus.fi, juhana.frosen@hus.fi, uha.hernesniemi@hus.fi \& E-mail addresses.reza.dashti@hus.fi, rdashti@istanbul.edu.tr \& E-mail address.aarno@sanger.ac.uk. 
and the integrity and remodeling of the wall of already established blood vessels [2]. Because of its role in both the development of blood vessels and the maintenance and repair of mature blood vessels, endoglin and other genes in this pathway are superb candidates for common forms of vascular anomalies, such as cerebral aneurysms. Mutations in this gene cause Mendelian vascular anomalies, the hereditary hemorrhagic telangiectasia type 1 (OMIM 187300), and thus a relevant hypothesis is that common variants in these genes could contribute to common forms of vascular anomalies.

The report of Sung-Pil Joo et al adds to a series of investigations which aim to resolve the potential link between endoglin and the genetic susceptibility to SAH and other cerebral vascular anomalies [1]. Previously, association with variants in the endoglin gene has been suggested in Japanese patients with aneurysmal SAH and in North American arteriovenous malformations $[10,13]$. However, subsequent studies have failed to replicate these original findings $[4,5,9,11,12]$. The Sung-Pil Joo et al study also fails to replicate the originally suggested variant in the endoglin gene, but provides suggestive evidence for association of another, nonsynonymous variant. An association with a variant which changes an amino acid is intriguing and potentially functionally relevant.

The somewhat conflicting results from the current and previous endoglin association studies reflect a relatively typical story. The story does not paint a clear picture of possible susceptibility variants and their potential impact for the trait. Why do different reports give conflicting results? Possible contributions to the inconsistency are population differences, differences in ascertainment schemes, differences in the diagnostic heterogeneity, and testing of different variants within the same gene. However, a major challenge has been the use of small study samples to test for association.

Frustrated by the inconsistency and slow progress of identification of true susceptibility variants in the field of common traits, international consortia with the help of major funding organizations and availability of new technology have changed the field. The field of research of genetic susceptibility to common traits has made a major leap during the last 18 months. Recent large genome-wide association studies testing hundreds of thousands of common variants involving thousands of cases and controls in several common traits (eg, type II diabetes, Crohn's disease, age-related macular degeneration) have provided important new knowledge for our future thinking on how genetic susceptibility to common traits should be tackled $[6,8,14]$. These new studies have identified tens of variants associated with several traits and pinpointed towards new, previously unpredicted cellular pathways. One of the lessons is that, typically, each individual variant has a very low impact on the disease susceptibility (relative risk 1.1-1.7). This low risk ratio has major impacts on study designs aiming at identifying gene variants associated with common traits, such as cerebral aneurysms. The identification of low impact variants requires very large sample sizes. For most complex traits the initial analysis should include around 2000 cases and preferentially more controls and replication studies, and thousands or even tens of thousands of cases and controls.

The need for very large sample sizes required for identification and verification of underlying predisposing gene variants has quickly changed our tradition of doing research. Such projects cannot be performed by one group, not even by one institution, and very rarely even by one nation. No clinic or institution can both possess sample collections of sufficient size and have the high throughput technological and analytical resources needed for these projects, yet not provide validation samples from different populations to evaluate the impact of identified variants in different populations. It is likely that many (if not most) central nervous system disorders will require even larger sample sizes than metabolic disorders which have championed the most recent successes in susceptibility gene 
identification. Thus, new ways to collaborate and invest in high throughput infrastructures to proceed in uncovering disease mechanisms by genetics and epidemiological genetics need to be adapted. This type of transition in how research goals are achieved was done in physics decades ago; we are still in the early stages of this transition.

As the evolution of totally noninvasive imaging techniques (magnetic resonance angiography and computed tomography angiography) and the tendency to perform more and more imaging studies of the cerebral vasculature seem to lead to an increased incidence of diagnosed unruptured aneurysms, we are often conferred with the clinical challenge of weighing the risks of operative or endovascular treatment against the rupture risk of the particular aneurysm. The increasing knowledge of the molecular pathology of aneurysm rupture will be needed to improve our abilities to predict rupture risk that is the sum of the complex interaction of patient and aneurysm-related anatomical factors, of genetic polymorphisms and background, and of exposure to various environmental factors. Research on genetic aberrations behind either sporadic or familial forms of cerebral aneurysms in different populations will help us to identify multiple genes that have key roles in the formation, maintenance, or repair of the cerebral vasculature. However, much work is still needed for us to really understand these complex mechanisms that are affected also by environmental factors and hemodynamics.

\section{References}

[1]. Alberts MJ, Davis JP, Graffagnino C, et al. Endoglin gene polymorphism as a risk factor for sporadic intracerebral hemorrhage. Ann Neurol. 1997; 41(5):683-6. [PubMed: 9153532]

[2]. Bobik A. Transforming growth factor-betas and vascular disorders. Arterioscler Thromb Vasc Biol. 2006; 26(8):1712-20. [PubMed: 16675726]

[3]. Helgadottir A, Thorleifsson G, Magnusson KP, et al. The same sequence variant on 9p21 associates with myocardial infarction, abdominal aortic aneurysm and intracranial aneurysm. Nat Gen. 2008; 40(2):217-24.

[4]. Krex D, Ziegler A, Schackert HK, Schackert G. Lack of association between endoglin intron 7 insertion polymorphism and intracranial aneurysms in a white population: evidence of racial/ ethnic differences. Stroke. 2001; 32(11):2689-94. [PubMed: 11692035]

[5]. Krex D, Schackert HK, Schackert G. Genesis of cerebral aneurysms-an update. Acta Neurochir (Wien). 2001; 143(5):429-48. [PubMed: 11482693]

[6]. Lango H, Weedon MN. What will whole genome searches for susceptibility genes for common complex disease offer to clinical practice? J Int Med. 2008; 263(1):16-42.

[7]. Lebrin F, Deckers M, Bertolino P, Ten Dijke P. TGF-beta receptor function in the endothelium. Cardiovasc Res. 2005; 65(3):599-608. [PubMed: 15664386]

[8]. Mathew CG. New links to the pathogenesis of Crohn disease provided by genome-wide association scans. Nature Rev Gen. 2008; 9(1):9-14.

[9]. Onda H, Kasuya H, Yoneyama T, et al. Endoglin is not a major susceptibility gene for intracranial aneurysm among Japanese. Stroke. 2003; 34(7):1640-4. [PubMed: 12775886]

[10]. Pawlikowska L, Tran MN, Achrol AS, et al. UCSF BAVM Study Project. Polymorphisms in transforming growth factor-beta-related genes ALK1 and ENG are associated with sporadic brain arteriovenous malformations. Stroke. 2005; 36(10):2278-80. [PubMed: 16179574]

[11]. Pera J, Slowik A, Dziedzic T, et al. Endoglin gene insertion polymorphism not associated with aneurysmal subarachnoid hemorrhage. J Neurosurg. 2005; 102(5):879-81. [PubMed: 15926713]

[12]. Peters DG, Kassam AB, Chang YF. A DNA sequence polymorphism in the endoglin gene is not associated with intracranial aneurysm or aneurysmal subarachnoid hemorrhage. Cerebrovasc Dis. 2005; 20(2):96-100. [PubMed: 15976502]

[13]. Takenaka K, Sakai H, Yamakawa H, et al. Polymorphism of the endoglin gene in patients with intracranial saccular aneurysms. J Neurosurg. 1999; 90(5):935-8. [PubMed: 10223461] 
[14]. Swaroop A, Branham KE, Chen W, Abescasis G. Genetic susceptibility to age relater macular degeneration: a paradigm for dissecting complex disease traits. Hum Mol Gen. 2007; 16(17):R174-82. [PubMed: 17911160]

[15]. van der Voet M, Olson JM, Kuivaniemi H, et al. Intracranial aneurysms in Finnish families: confirmation of linkage and refinement of the interval to chromosome 19q13.3. Am J Hum Gen. 2004; 74:564-71.

[16]. Wills S, Ronkainen A, van der Voet M, et al. Familial intracranial aneurysms: an analysis of 346 multiplex Finnish families. Stroke. 2003; 34:1370-4. [PubMed: 12750547] 